\title{
Strates
}

STRATES Matériaux pour la recherche en sciences sociales

Hors-série | 2002

Parcours dans la recherche urbaine, Michel Rochefort, un géographe engagé

\section{Les notions de réseau urbain et d'armature urbaine}

\section{Michel Rochefort}

\section{OpenEdition}

Journals

Édition électronique

URL : http://journals.openedition.org/strates/512

DOI : 10.4000/strates.512

ISSN : 1777-5442

Éditeur

Laboratoire Ladyss

Édition imprimée

Date de publication : 1 janvier 2002

ISSN : 0768-8067

Référence électronique

Michel Rochefort, «Les notions de réseau urbain et d'armature urbaine », Strates [En ligne], Hors-série | 2002, mis en ligne le 02 mai 2005, consulté le 08 septembre 2020. URL : http://

journals.openedition.org/strates/512 ; DOI : https://doi.org/10.4000/strates.512

Ce document a été généré automatiquement le 8 septembre 2020

Tous droits réservés 


\title{
Les notions de réseau urbain et d'armature urbaine
}

\author{
Michel Rochefort
}

1 C'est aujourd'hui une vérité d'évidence que les villes, considérées dans leurs fonctions tertiaires comme centres des activités de service, ne sont pas des organismes indépendants et isolés les uns des autres. L'espace ne se découpe pas en zones simples et autonomes commandées par un centre urbain doté de tous les équipements nécessaires à la vie de cette portion d'espace. Selon leur nature, les services se localisent dans diverses villes plus ou moins grandes et chaque point de l'espace dépend de l'un ou l'autre de ces centres selon le service auquel il doit avoir recours. L'unité réelle d'organisation est donc constituée par l'ensemble des centres nécessaires pour fournir la totalité des services que réclament les activités économiques et la vie de la population. L'étude de cette réalité géographique repose par conséquent d'abord sur l'analyse des types de centres de services et de leurs zones respectives d'influence, en les saisissant dans leurs rapports réciproques, puis sur l'organisation de l'espace qui en résulte du double point de vue de la localisation des différents centres et du découpage de l'espace en zones organisées.

2 Dans cette optique, on peut se limiter à l'étude d'une "région ", c'est-à-dire à l'espace délimité par l'influence d'une grande ville, dotée d'une gamme suffisante de services pour que les habitants de la zone puissent éviter tout recours généralisé à une autre ville plus importante et mieux équipée qu'elle. Cette «métropole régionale » organise la vie économique et sociale de sa région soit directement par suite des services qu'elle seule possède dans cette portion d'espace, soit indirectement lorsqu'elle renferme le niveau de commandement de services hiérarchisés dont les relais se trouvent répartis dans d'autres villes plus modestes situées à l'intérieur de sa région. Chacune de ces dernières d'autre part possède une certaine gamme de services plus courants; la région se divise de ce point de vue en diverses portions d'espace qui correspondent aux zones d'influence de ces centres urbains secondaires.

3 Cependant, dans la plupart des pays, les métropoles régionales dépendent à leur tour d'un certain nombre de services centraux localisés dans la capitale nationale. On doit 
donc concevoir aussi une étude à l'échelle de la nation pour définir les rapports entre la capitale et les centres régionaux et pour décrire le découpage de l'espace qui en résulte.

Peut-on proposer d'appeler réseau urbain l'organisation des centres urbains et de leurs zones d'influence à l'intérieur d'une région commandée par un centre régional et armature urbaine l'organisation de ces centres régionaux à l'intérieur de l'espace commandé par la capitale nationale?

Que l'étude se place à l'une ou l'autre de ces deux échelles spatiales la différenciation des centres urbains repose sur des causes de nature variée et les éléments du réseau urbain, constitués par les divers types de centre sont plus ou moins nombreux selon le mode de formation de celui-ci.

I. facteurs de différenciation des types de centres

6 Les centres de service qui constituent un réseau ou une armature urbaine se différencient d'abord par la nature des services qu'ils possèdent, sans que cela implique une véritable dépendance des uns vis-à-vis des autres; ils se différencient aussi par la place qu'ils occupent dans la structure de certains services caractérisés par une hiérarchie interne.

1. Différenciation par la nature des services

7 Dans une région, les centres urbains secondaires ne possèdent, par exemple, que les commerces les plus courants. Lorsqu'un habitant de ces petites villes ou de leur zone d'influence a besoin d'un objet moins usuel, il doit avoir recours aux services d'une cité plus importante qui dispose d'un tel commerce. La différenciation des centres repose uniquement sur la plus ou moins grande fréquence du recours au service, qui implique une plus ou moins grande " rareté » de celui-ci dans l'espace régional, par suite de la nécessaire rentabilité de sa localisation. Selon le nombre de recours par an et le pourcentage des habitants qui effectuent ce recours, on peut définir le degré des services, chacun de ceux-ci nécessitant une certaine masse globale de population pour qu'il dispose d'une clientèle suffisante pour être rentable. Cette masse de population varie évidemment pour un même service selon les structures socio-professionnelles et les niveaux de vie des habitants. La différenciation des centres d'un réseau repose donc de ce point de vue sur des niveaux de services définis par les divers degrés de rareté de ceux-ci. La dépendance des centres secondaires vis-à-vis des centres principaux est due simplement à l'absence de certains services qui contraint des petites villes lorsqu'ils ont besoin de ceux-ci à avoir recours à la grande ville voisine.

\section{Différenciation par la structure des services}

8 Dans d'autres domaines, au contraire, la dépendance des échelons inférieurs du réseau par rapport à l'échelon supérieur repose sur la structure interne du service. Il peut s'agir d'une dépendance rigide due par exemple à l'organisation administrative de la nation et définie par la localisation dans les diverses villes des différents échelons de la hiérarchie administrative. Il peut s'agir au contraire d'une dépendance plus dynamique, reposant sur la structure financière des activités tertiaires du secteur privé: l'organisation des grandes banques, avec les différents échelons de leur direction générale, de leurs directions régionales, de leurs agences et de leurs guichets en fournit un exemple. La ville qui possède une direction régionale commande aux villes qui ne possèdent que des agences dépendant de celles-ci.

II. Variétés des éléments d'un réseau ou d'une armature urbaine

9 On pourrait imaginer, en théorie, un schéma général de réseau urbain avec des éléments correspondant aux divers niveaux des services autonomes et aux divers 
degrés des services à structure interne hiérarchisée. On constate, en fait, selon les pays et selon les régions à l'intérieur de chaque pays, une extrême diversité. La fonction de capitale nationale elle-même, prend des aspects différents selon que la structure du pays est centraliste ou fédéraliste; elle peut être groupée dans une ville ou partagée entre plusieurs cités. Tantôt les métropoles régionales s'affirment comme les centres majeurs de la vie de relations, cas de l'Allemagne, tantôt elles se subordonnent étroitement à la capitale nationale, cas de la France. Quant aux échelons inférieurs, ils varient à l'infini en nombre et en qualité. On peut pourtant retenir de façon très générale quelques grandes catégories de centres qui ont déjà été décrites par plusieurs auteurs et en particulier par J. Labasse: le centre local à recours journalier ou hebdomadaire, le centre moyen à recours mensuel, le centre régional dont la fonction essentielle ne correspond plus à un recours direct mais à un commandement des services fournis par les centres inférieurs; la capitale nationale, centre de fonction de direction.

On pourrait imaginer de même une répartition géométrique de ces divers types de centres à l'intérieur de l'espace desservi par un réseau urbain, mais dans ce domaine, la réalité est encore beaucoup plus éloignée d'un tel schéma et la position des différents éléments des réseaux urbains relève de facteurs variés et complexes qui ne se plient guère à une généralisation systématique, pas plus que la forme de leurs respectives zones d'influence. Face à cette variété des éléments des réseaux urbains, nous nous contenterons ici de quelques suggestions sur les grands facteurs de cette diversité et de l'analyse d'un cas : celui des centres de niveau régional en France.

1. Facteurs généraux de diversité

11 a. Les uns relèvent des caractères actuels des pays ou des régions. Il semble, d'une façon générale, que les pays ou régions à bas niveau de vie soient caractérisés par une nette déficience des échelons intermédiaires : la masse pauvre de la population ne peut guère avoir recours qu'aux services les plus courants, fournis par le centre élémentaire le plus proche, la portion riche habite dans la grande ville et utilise directement tous les services de celle-ci qui renferme aussi les organismes de direction de l'économie régionale ; il y a peu de place pour des villes moyennes.

$12 \mathrm{Au}$ contraire, dans les pays à haut niveau de vie, les échelons intermédiaires se multiplient, sans qu'il existe toujours entre eux une hiérarchie nette ou une dépendance précise des uns vis-à-vis des autres. Cette richesse des réseaux en divers types de centres traduit avant tout la multiplicité des services utilisés par les activités économiques et par les particuliers en pays développés; aussi varie-t-elle, dans un même pays, selon que domine l'activité industrielle ou l'activité agricole, selon que la densité de population est plus ou moins forte et la fraction aisée de cette population plus nombreuse. Cette richesse pourtant semble parfois dépasser les besoins actuels; elle est alors due à des facteurs historiques.

b. Les modalités de l'élaboration des réseaux pèsent en effet d'un poids très lourd dans l'explication de leurs caractères actuels. D'une façon très générale, les réseaux urbains se sont façonnés à partir du moment où les transports modernes ont facilité les déplacements de relations ; ils ont, dans la plupart des cas, commencé à s'élaborer au $\mathrm{XIX}^{\mathrm{e}}$ siècle, sauf dans les zones où l'occupation humaine est très récente. Ils ont subi une évolution complexe, à partir de leur création; ils ont dû enregistrer l'évolution même des activités de services dont les villes sont le support: modification des structures administratives, concentration financière des activités économiques, 
développement des besoins en services lié à l'essor des niveaux de vie et des civilisations. Ils ont aussi subi tous les contrecoups des changements dans les techniques de transport et ont dû se réorganiser en fonction de l'amenuisement progressif des distances.

Bien plus, ces réseaux urbains sont nés la plupart du temps à partir d'un semis de villes anciennes, préalables à la Révolution industrielle, qui ont plus ou moins facilement acquis les services et équipements qui se sont multipliés à partir de cette époque, sans occuper toujours au sein de l'espace les positions les plus appropriées pour exercer ces nouvelles fonctions. Bref, les aspects actuels des réseaux ne traduisent pas seulement les besoins actuels, en eux-mêmes variés, des régions et des pays, mais aussi des phénomènes de survivance des phases antérieures de l'implantation des villes. La complexité qui en résulte peut être illustrée par la simple analyse des caractères actuels du niveau régional de l'armature urbaine française.

2. Un exemple de complexité : les villes de niveau régional dans l'armature urbaine française

Cet aspect de l'armature urbaine française a été étudié, depuis plusieurs années sous la direction de M. Hautreux et de l'auteur de cette communication dans le cadre des recherches pour l'aménagement du territoire [22]. On s'est efforcé d'identifier toutes les villes qui exercent dans la vie de relations de leur région un rôle polarisateur majeur ou simplement partiel, ceci par opposition aux villes dont la fonction et l'influence régionales sont inexistantes ou en tout cas négligeables. La méthode en a été exposée lors du colloque franco-polonais de juin 1963, à Paris.

us nous contenterons donc ici d'exposer rapidement les principaux résultats en insistant sur la complexité de la réalité qu'ils font apparaître. On s'aperçoit d'abord qu'au sein de toutes les villes de France qui dans un domaine ou dans un autre exercent une fonction d'encadrement de niveau régional, il existe des différences sensibles qui permettent de les diviser en plusieurs catégories. Certaines, particulièrement bien équipées dans tous les domaines des différents services nécessaires à la vie régionale, constituent un premier ensemble de huit villes qu'on peut considérer comme les métropoles régionales dans l'armature urbaine française. Ce sont les villes de Lyon, Marseille, Bordeaux, Lille, Toulouse, Strasbourg, Nantes et Nancy. Remarquons que même dans cette catégorie, il y a une différence très nette dans la puissance du rôle régional entre des villes comme Lyon ou Marseille, supérieures à la moyenne et des villes comme Nantes et Nancy déjà frappées d'une relative faiblesse de certains équipements. Pourtant la différence est sensible entre la moins bien équipée de ces métropoles et la mieux armée des villes du deuxième ensemble qui comprend dix villes, celles de Grenoble, Nice, Clermont-Ferrand, Rouen, Dijon, Montpellier, Saint-Etienne, Caen, Limoges et Rennes. Ce deuxième groupe que nous appellerons celui des centres régionaux de plein exercice est suivi par une série de 24 villes qui participent encore à un rôle à l'échelle d'un espace régional, mais sont frappées d'une faiblesse radicale dans l'une ou l'autre des différentes catégories de services de ce niveau ; nous les appellerons les villes à fonction régionale incomplète.

On voit donc que dans cette catégorie de villes qui constituent le niveau régional de l'armature urbaine, il y a des différences considérables d'équipements et qu'une analyse précise contraint à faire éclater cette catégorie en plusieurs sous-groupes. En examinant la figure 1 , on peut constater que ces différents types de centres régionaux occupent des positions assez diverses au sein de l'espace français. 


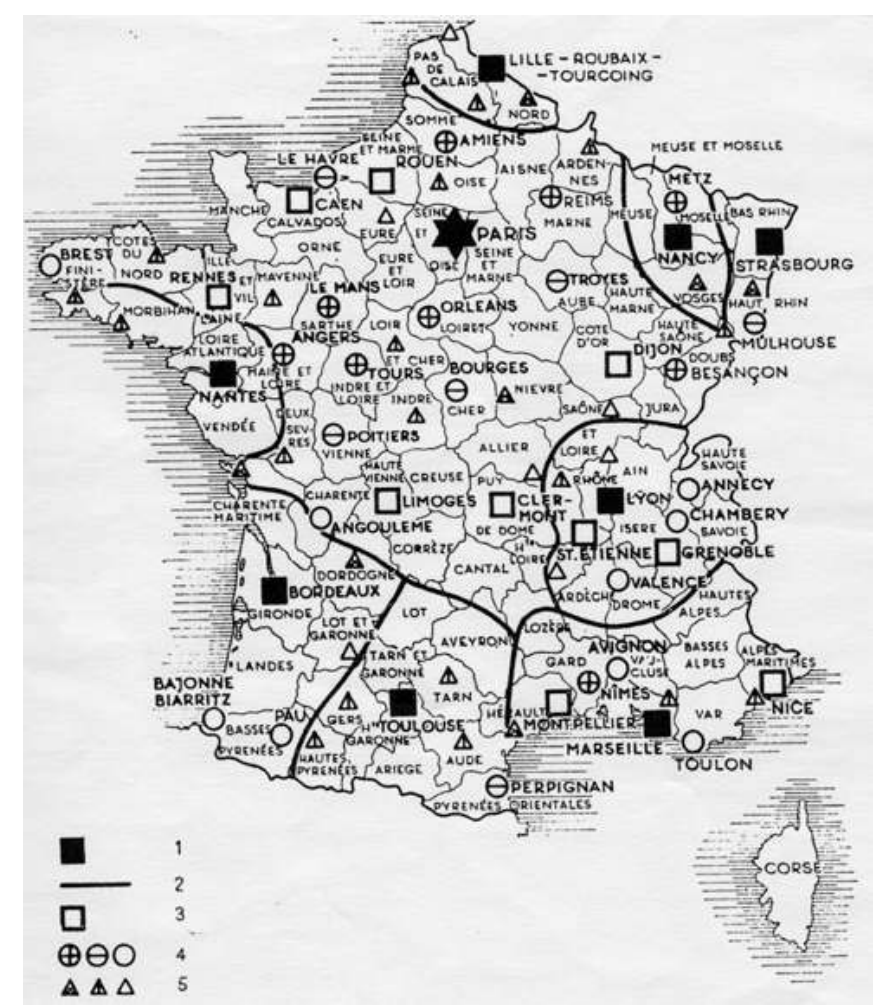

Légende de la figure 1

Fonction régionale dans l'armature française ; localisation des villes appartenant actuellement au niveau régional

1. métropoles ; 2. limites actuelles des zones d'influence des métropoles ; 3 . centres régionaux ; 4. villes à fonction régionale incomplète ; 5 . villes se situant au niveau des chefs-lieux de département bien équipés (dans les deux dernières rubriques, les trois signes différents correspondent respectivement aux trois subdivisions distinguées dans le classement global).

On peut observer d'abord que les huit métropoles régionales ont une disposition nettement périphérique par rapport à l'espace français, c'est-à-dire qu'elles sont aussi éloignées que possible de la capitale nationale.

On peut donc estimer que celle-ci a entravé, dans un vaste rayon autour d'elle, le développement complet de véritables métropoles régionales et que la complexité des villes à fonction régionale relève de ce point de vue du rôle concurrentiel de la capitale nationale dans une vaste portion du territoire français.

Les centres régionaux de plein exercice et les villes à fonction régionale incomplète sont beaucoup plus diversement localisés. Certains se trouvent dans l'espace qui relève de l'influence de l'une ou l'autre des métropoles régionales, que ce soit Mulhouse dans la zone d'influence de Strasbourg, Metz dans celle de Nancy, Grenoble et Saint-Etienne dans celle de Lyon, Montpellier dans celle de Marseille et Perpignan dans celle de Toulouse.

On s'aperçoit très vite à l'analyse que ce rattachement est la plupart du temps illusoire et que ces centres régionaux, moins bien équipés que les métropoles ne sont pas hiérarchiquement subordonnés à celle-ci, sauf pour quelques services exceptionnels et qu'en général ils regardent directement vers Paris.

Dans le vaste secteur qui échappe à l'influence des métropoles et où par conséquent on doit considérer que la capitale remplit cette fonction, on note de nouveau que six centres régionaux de plein exercice qui y existent, se trouvent à la périphérie de cet 
espace ainsi délimité. Au contraire, des villes à fonction régionale incomplète, comme Amiens, Reims, Troyes ou Orléans semblent constituer une deuxième auréole plus proche de Paris. Ici encore, il faut se méfier de tout géométrisme : on trouve également des villes à fonction régionale incomplète au-delà des centres régionaux - tel est le cas de Besançon par rapport à Dijon - et on trouve aussi dans les pays de la Loire une accumulation de villes à fonction régionale incomplète, sans que l'une ait une influence appréciable sur les autres, toutes se rattachant en fait directement à Paris.

$\mathrm{Au}$ terme de ce rapide tour d'horizon, on peut constater qu'en fait il n'y a pas de véritable organisation hiérarchisée entre les trois degrés des centres d'encadrement tertiaire du niveau régional. En d'autres termes, les usagers n'ont pas successivement recours, au fur et à mesure qu'il s'agit de services plus rares, à une ville à fonction régionale incomplète puis à un centre régional de plein exercice et enfin à une métropole régionale.

Il est rare en effet que les centres régionaux des degrés subalternes soient situés à l'intérieur des zones d'influence des villes du groupe supérieur : les centres régionaux les mieux équipés après les métropoles se rencontrent pour la plupart, soit dans les zones proches de la capitale où le puissant rayonnement de celle-ci interdit à toute cité d'atteindre au niveau supérieur, soit dans des régions excentriques trop peu peuplées et trop peu actives pour qu'une métropole régionale s'y soit développée. D'autre part, les centres régionaux subalternes qui s'insèrent dans les zones d'influence des métropoles régionales n'en dépendent pas d'une manière absolue et entretiennent surtout des rapports directs avec Paris. Certes, on peut bien déceler quelques liens de complémentarité correspondant à une hiérarchie, mais celle-ci est loin d'être rigoureuse et les relations par lesquelles elle se traduit sont souvent moins importantes que celles qui relient les différentes cités en question directement à la capitale nationale.

On peut donc conclure que l'armature urbaine française est caractérisée par l'existence d'un ensemble d'agglomérations de niveau régional diversifiées quant à la qualité et la variété de leurs services. Ces différences s'expliquent, la plupart du temps, par le dynamisme propre de chaque ville, donc par son histoire, par les besoins de ses habitants, de ses activités de production aussi bien que par les besoins d'une zone d'influence plus ou moins vaste, beaucoup plus que par une organisation systématique des relations de dépendance reposant sur le recours, pour les moins équipées, au service des mieux équipées.

Ainsi, l'organisation spontanée de l'encadrement tertiaire de la France n'a pas abouti à la formation d'une armature urbaine systématiquement ordonnée, constituée par plusieurs catégories de centres hiérarchisés en fonction des degrés de rareté des équipements ou des échelons du pouvoir de décision. La complexité de l'évolution économique et sociale de la France est partiellement responsable de cette absence de schématisation dans les phénomènes d'armature et de réseau urbain, mais il est sans doute difficile de trouver des exemples de réseau urbain schématique même dans des pays d'évolution plus simple. Presque toujours en effet, les villes qui sont sensibles aussi bien à tous les caractères actuels des systèmes économiques et sociaux qu'au poids du passé dû aux phases antérieures d'organisation de l'espace sont des organismes trop riches pour que l'un puisse être exactement semblable à l'autre même en isolant de leurs activités celles qui correspondent à leurs fonctions d'encadrement tertiaire du pays. 
Il ne faut certes pas nier la réalité des notions de réseaux urbains et d'armature urbaine malgré la complexité dont on vient d'esquisser un exemple. Mais il faut considérer que c'est une réalité de structure. Un pays ou une région, au moins en économie développée, possède toujours une armature et un réseau urbain de même qu'une maison possède toujours une charpente ; mais il y a une infinie variété de charpentes selon les maisons.

D'autre part, réseaux et armature sont la plupart du temps affectés de phénomènes de survivance dus à la permanence de toute ville, même après qu'aient disparu les facteurs qui furent à l'origine de sa création. Réseaux et armatures urbaines paraissent parfois inadéquats et désadaptés par rapport aux besoins actuels. Aussi, se pose le problème de l'aménagement volontaire de l'armature et des réseaux urbains d'un pays dès que celuici se propose de lancer une politique cohérente d'aménagement du territoire.

\section{BIBLIOGRAPHIE}

[1] Beaujeu-Garnier J., Chabot G., Traité de géographie urbaine, Paris, 1963.

[2] Beckmann M., City Hierarchies and the Distribution of City Size ; Structural Proportions in a Hierarchy of a City, Economic Development and Cultural Change, 1958.

[3] Berry B. L., The Functional Bases of the Central Place Hierarchy, Econ. Geogr., 1956.

[4] Id. Note on Hierarchy of Central Place, Econ. Geogr., 1958.

[5] Id. Recent Development of Central Place Theory, PAP. RSA, 1958.

[6] Bonetti E., La teoria della località centrale, Trieste, 1964.

[7] Bracey H. E., A rural component of centrality applied to six southern countries in the UK, Econ. Geogr., 1956.

[8] Carter H., Urban Grades and Spheres of Influence in SW Wales, Scottish Geogr. Mag., 1955.

[9] Id. The Urban Hierarchy and Historical Geography, Geogr. Studies, 1956.

[10] Chabot G., Les villes, aperçu de géographie humaine, Paris, 1948.

[11] Christaller W., Das Problem der zentralen Orte, Jena, 1933.

[12] Coppolani J., Le réseau urbain de la France, sa structure et son aménagement, Paris, 1959.

[13] CRU (Centre de Recherche d'Urbanisme), L'Urbanisation française, Paris, 1964.

[14] Dickinson R. E., City Region and Regionalism, London, 1947.

[15] Dugrand R., Villes et campagnes en Bas-Languedoc. Le réseau urbain du Bas-Languedoc méditerranéen, Paris, 1963.

[16] George P., La ville - le fait urbain à travers le monde, Paris, 1954.

[17] George P., Précis de géographie urbaine, Paris, 1961. 
[18] George P., Deux études de réseaux urbains : l'Alsace et Bas-Languedoc d'après M. Rochefort et R. Dugrand, Ann. Geogr., 1964, 396.

[19] George P., Présentation de l'armature urbaine française, Humanisme et Entreprise, 1965, 4.

[20] Hautreux J., Rochefort M., Le niveau supérieur de l'armature urbaine française, Paris, 1963.

[21] Hautreux J., Les principales villes attractives et leur ressort d'influence, Revue d'urbanisme, $1963,78$.

[22] Hautreux J., Rochefort M., Métropole et centres régionaux dans l'armature urbaine française, Construction - Aménagement, 1964, 17.

[23] Id. La fonction régionale dans l'armature urbaine française, min. Constr., 1964, 4.

[24] Id. Physionomie générale de l'armature urbaine française, Ann. Géogr., 1964.

[25] Juillard E., Essai de hiérarchisation des centres urbains actuels, Min. constr., CEES, 1961.

[26] Kayser B., Campagnes et villes de la Côte d'Azur ; essai sur les conséquences du développement urbain, Monaco, 1958.

[27] Labasse J., Les capitaux et la région, Paris, 1955.

[28] Labasse J., Rochefort M., Le rôle des équipements tertiaires supérieurs dans la polarisation de la vie régionale en Europe Occidentale, Min. Constr., 1964.

[29] Neef E., Das Problem der zentralen Orte, Petermanns Geogr. Mitt., 95, 1951.

[30] Philbrick A. K., Areal Functional Organization in Regional Geography, PAP. R.S.A., 1957.

[31] Prost M. A., La hiérarchie des villes en fonction de leurs activités de commerce et de services, Paris, 1965.

[32] Piatier A., L'attraction commerciale des villes ; une nouvelle méthode de mesure, Rev. Jurid. et écon. du Sud Ouest, 4, 1956.

[33] Reilly W. J., Methods for the study of retail relationships, Univ. of Texas Bul., 2944, 1929.

[34] Id. The Law of Retail Gravitation, New-York, 1931.

[35] Rochefort M., L'organisation urbaine de l'Alsace, Paris, 1960.

[36] Schultze J., Zur Ammerdbarkeit der Theorie der zentralen Orte, Petermanns Geogr. Mitt., 95, 1951.

[37] Smailes A. E., The Urban Hierarchy in England and Wales, Geography, 29, 1944.

\section{NOTES}

1. Geographia Polonica 12, 1967, p. 19-28 